\title{
Quantum Spin-Hall Effect and Topologically Invariant Chern Numbers
}

\author{
D. N. Sheng, ${ }^{1}$ Z. Y. Weng, ${ }^{2}$ L. Sheng, ${ }^{3}$ and F. D. M. Haldane ${ }^{4}$ \\ ${ }^{1}$ Department of Physics and Astronomy, California State University, Northridge, California 91330, USA \\ ${ }^{2}$ Center for Advanced Study, Tsinghua University, Beijing 100084, China \\ ${ }^{3}$ Department of Physics and Texas Center for Superconductivity, University of Houston, Houston, Texas 77204, USA \\ ${ }^{4}$ Department of Physics, Princeton University, Princeton, New Jersey 08544, USA
}

(Received 6 March 2006; published 21 July 2006)

\begin{abstract}
We present a topological description of the quantum spin-Hall effect (QSHE) in a two-dimensional electron system on a honeycomb lattice with both intrinsic and Rashba spin-orbit couplings. We show that the topology of the band insulator can be characterized by a $2 \times 2$ matrix of first Chern integers. The nontrivial QSHE phase is identified by the nonzero diagonal matrix elements of the Chern number matrix (CNM). A spin Chern number is derived from the CNM, which is conserved in the presence of finite disorder scattering and spin nonconserving Rashba coupling. By using the Laughlin gedanken experiment, we numerically calculate the spin polarization and spin transfer rate of the conducting edge states and determine a phase diagram for the QSHE.
\end{abstract}

DOI: 10.1103/PhysRevLett.97.036808

Topological quantities are fundamentally important in characterizing the transverse electrical transport property in integer and fractional quantum Hall effect states [1,2] of two-dimensional (2D) electron systems. It was first revealed by Thouless et. al. [3] that each integer quantum Hall effect (IQHE) state is associated with a topologically invariant integer known as the first Chern number, which precisely equals the Hall conductance in units of $e^{2} / h$. The exact quantization of the Hall conductance can also be formulated in terms of a $2 \mathrm{D}$ band-structure Berry phase [3-6], which remains an integral invariant till the band energy gap (or the mobility gap [7] in the presence of disorder) collapses.

While the conventional IQHE is usually associated with strong magnetic fields, Haldane [8] has explicitly shown that it can actually occur in the absence of magnetic field in band insulators with graphenelike band structure. The onecomponent Haldane model explicitly breaks time-reversal symmetry, resulting in a condensed-matter realization of a parity symmetry anomaly with chiral edge states at the boundary of the sample. In realistic electron systems, however, the coupled spin degrees of freedom can recover the time-reversal symmetry by forming Kramers degenerate states, which belong to the universality class of zero charge Chern number as the total Berry curvature of the occupied energy band of both spins sums to zero.

This class of insulators has been recently found $[9,10]$ to possess a dissipationless quantum spin-Hall effect (QSHE) [11], which is distinct from the intrinsic spin-Hall effect in the metallic systems [12]. The QSHE has been shown to be robust against disorder scattering and other perturbation effects $[9,10]$. Whether there exists an underlying topological invariant "protecting" the QSHE is a very important issue for both fundamental understanding and potential applications of the QSHE. While the previously proposed $[9,13] Z_{2}$ classification of the QSHE suggests that the conducting edge states are protected by time-
PACS numbers: 73.43.Nq, 11.15.- q, 72.25.-b

reversal symmetry, it does not distinguish between two QSHE states with spin-Hall conductance (SHC) of opposite signs. Thus it remains an open issue if the QSHE states can be classified by a more definite topological quantum number similar to the Chern number for the conventional IQHE.

In this Letter, we clarify the topological nature of the QSHE in the graphene model with spin-orbit coupling. We present numerical evidence that a pair of nonchiral conducting edge states is responsible for the dissipationless spin current in the open system with disorder scattering. The spin transfer and spin accumulation are calculated, which increase linearly with the flux insertion in the Laughlin gedanken experiment [14]. We further establish the relation between the conducting edge states, the topological invariant Chern number matrix (CNM), and a spin Chern number for the bulk system with electron Fermi energy lying inside the band gap. The nontrivial spin Chern number distinguishes a QSHE state from an ordinary band insulating state and is responsible for the robust quantum SHC.

We begin with the 2D honeycomb lattice model [8-10], which is relevant to the $2 \mathrm{D}$ electrons in a single-atomic layer graphene system [15]:

$$
\begin{aligned}
H_{0}= & -t \sum_{\langle i j\rangle} c_{i}^{\dagger} c_{j}+\frac{2 i}{\sqrt{3}} V_{\mathrm{SO}} \sum_{\langle\langle i j\rangle\rangle} c_{i}^{\dagger} \boldsymbol{\sigma} \cdot\left(\mathbf{d}_{k j} \times \mathbf{d}_{i k}\right) c_{j} \\
& +i V_{R} \sum_{\langle i j\rangle} c_{i}^{\dagger} \hat{\mathbf{z}} \cdot\left(\boldsymbol{\sigma} \times \mathbf{d}_{i j}\right) c_{j}+\sum_{i} w_{i} c_{i}^{\dagger} c_{i},
\end{aligned}
$$

where $c_{i}^{\dagger}=\left(c_{i \dagger}^{\dagger}, c_{i \downarrow}^{\dagger}\right)$ are electron creation operators and $\boldsymbol{\sigma}$ is the Pauli matrix. The first term is the usual nearest neighbor hopping term, and the second term is an intrinsic SO coupling preserving the lattice symmetries with $i$ and $j$ as two next nearest neighbor sites and $k$ their unique common nearest neighbor. Here, the vector $\mathbf{d}_{i k}$ points from $k$ to $i$, with the distance between two nearest neighbor 
sites taken to be unity. The third term stands for the Rashba SO coupling with strength $V_{R}$, and the last term represents an on-site random disorder with strength $\left|w_{i}\right| \leq W / 2$.

In the absence of the Rashba SO coupling $V_{R}=0$, the model Eq. (1) reduces to a two-component Haldane model [8], which exhibits a quantized SHC $\sigma_{s H}= \pm 2 \frac{e}{4 \pi}$ with sign depending on the sign of $V_{\mathrm{SO}}$. In the following, we will perform systematic numerical simulations designed to reveal the topological characteristics of such a nontrivial band insulator in the general case of finite $V_{R}$ with disorder scattering.

Gauge experiment and edge states free from backward scattering. - Let us first study a rectangular geometry of a honeycomb lattice consisting of $N_{y}$ zigzag chains with $N_{x}$ atom sites on each chain. A twisted boundary condition is imposed [16] in the $\hat{\mathbf{y}}$ direction, and an open boundary condition is used in the $\hat{\mathbf{x}}$ direction. Since the boundary phase $\theta_{y}$ does not depend on spin, the system is also equivalent to a cylindric geometry threaded by a flux $\Phi=$ $\frac{\theta_{y}}{2 \pi}$ in the axial direction ( $\hat{\mathbf{x}}$ direction), similar to that used in the Laughlin gedanken experiment [14] for IQHE.

We find that some new states emerge within the bulk energy gap $-0.1 t<E_{m}<0.2 t$ with the density of states much smaller than that of the bulk states outside the energy gap. These midgap states can be identified as edge states with their wave functions localized within a few lattice constants at the two open boundaries in the $\hat{\mathbf{x}}$ direction and extended along the $\hat{\mathbf{y}}$ direction. The edge states continuously evolve and cross each other with increasing $\theta_{y}$ as shown in Fig. 1 for $V_{\text {SO }}=0.05 t, V_{R}=0.05 t, W=1.0 t$, and system size $N \equiv N_{x} \times N_{y}=480 \times 240$. Similar to IQHE, the level crossing of the edge states in Fig. 1 clearly demonstrates the absence of backward scattering, and thus each state will not simply evolve back to its original state after the insertion of one flux quantum $\Phi=1$ (or $\theta_{y}=$ $2 \pi$ ), which leads to an adiabatic transverse transport of spins from one edge to the other [16]. For a Fermi energy $E_{f}$ inside the band gap, as indicated by the dashed line in Fig. 1, there is always a pair of edge states crossing the $E_{f}$

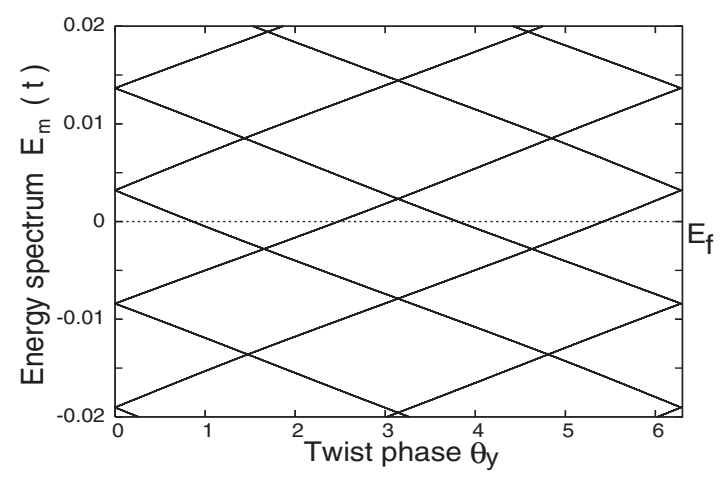

FIG. 1. The evolution of edge levels inside the bulk gap with twist boundary phase $\theta_{y}$ in a cylindric 2D sample of $N=480 \times$ $240, V_{\text {SO }}=0.05 t, V_{R}=0.05 t$, and $W=1.0 t$. line and evolving out of the Fermi sea. It can be identified in the real space as moving oppositely, either from the left edge to the right one or vice versa, and thus it does not contribute to a net transverse electric charge transport.

Robust spin polarization carried by the edge states. We then explicitly compute the spin polarization $P_{m}^{z}=$ $\frac{\hbar}{2}\left\langle m\left|\sum_{i} c_{i}^{+} \sigma^{z} c_{i}\right| m\right\rangle$ along the $\hat{\mathbf{z}}$ direction with $|m\rangle$ as the $m$ th single-particle eigenstate. Spin polarizations along the $\hat{\mathbf{x}}$ and $\hat{\mathbf{y}}$ directions are found to be much smaller. At $\theta_{y}=0$, all the eigenstates form Kramers degenerate pairs, and each pair carries totally zero spin due to time-reversal symmetry. We find that upon insertion of the flux $\left(\theta_{y} \neq 0\right)$, a nonzero spin polarization is quickly developed for each edge state. Figure 2 shows the spin polarization of each eigenstate as a function of eigenenergy $E_{m}$ at $\theta_{y}=2 \pi / 48$. The spin polarization is finite only for the edge states within the energy gap and insensitive to disorder configurations and sample sizes, as all the data points nicely collapse together. We find that $P_{m}^{z}$ is mainly affected by the Rashba coupling $V_{R}$ and decreases monotonically with increasing $V_{R}$.

Spin transfer rate and phase diagram. - Now we can determine the spin transfer by the edge states with the adiabatic insertion of the flux. There is always a pair of states crossing the $E_{f}=0$ line from below, as shown in Fig. 1. One state carries a positive $P_{m}^{z}$, which moves to the right edge of the sample, and the other with negative $P_{m}^{z}$ moves to the left edge, as determined from the wave functions. Thus after the insertion of one flux quantum $\left(\theta_{y}=0 \rightarrow 2 \pi\right)$, a net spin transfer occurs, which results in spin accumulations at the two open edges of the sample. We can determine the average spin transfer rate $\gamma$ by using the relation $\gamma=\frac{\Delta\left\langle S^{z}\right\rangle_{\text {edge }}}{\Delta \Phi}=\left.\left\langle S_{z}\right\rangle_{\text {edge }}\right|_{\theta=2 \pi}-\left.\left\langle S_{z}\right\rangle_{\text {edge }}\right|_{\theta=0}$ with $\left\langle S^{z}\right\rangle_{\text {edge }}=\sum_{m}^{\prime} P_{m}^{z}$, where the summation runs over only the edge states at the right edge. As shown in Fig. 3(a), the spin transfer rate remains about 1.8 (in units of $\frac{e}{4 \pi}$ ) till a critical disorder strength $W_{c} \sim 2.4 t$ is reached,

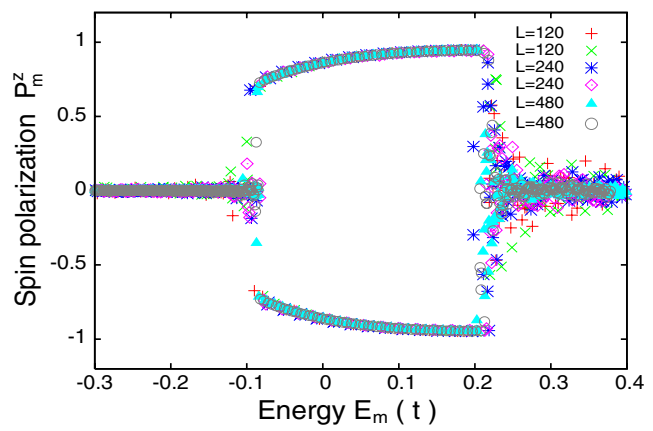

FIG. 2 (color online). Spin polarization $P_{m}^{z}$ defined in the text (in units of $\hbar / 2$ ) as a function of eigenenergy $E_{m}$ for two random disorder configurations (we have checked over 100 configurations) of strength $W=1.0 t$ at different system sizes $N=120 \times$ 120-480 $\times 480$ (with $N_{x}=N_{y}=L$ ). $V_{\text {SO }}$ and $V_{R}$ are the same as in Fig. 1. 

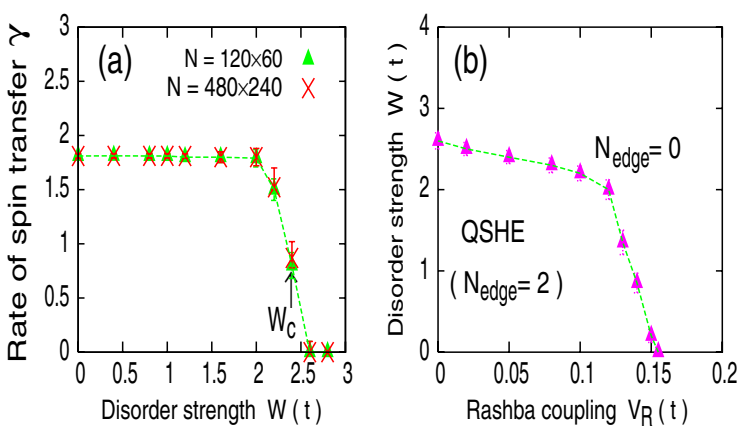

FIG. 3 (color online). (a) Spin transfer rate $\gamma=\frac{\Delta\left\langle S^{z}\right\rangle_{\text {edge }}}{\Delta \Phi}$ (in units of $e / 4 \pi)$ vs disorder strength $W$ at $V_{\mathrm{SO}}=0.05 t$ and $V_{R}=$ $0.05 t$ averaged over 500 disorder configurations. (b) The phase boundary for the QSHE determined from the spin transfer, where the transition happens with a change of the numbers of edge channels from $N_{\text {edge }}=2$ to $N_{\text {edge }}=0$.

beyond which $\gamma$ vanishes quickly. By analogy with IQHE [14], the spin transfer rate corresponds to a finite SHC of value around $1.8 \frac{e}{4 \pi}$, which is nonquantized as spins are nonconserved. By determining critical $W_{c}$ at different $V_{R}$ 's through the calculation of the spin transfer rate, we obtain a phase diagram in the parameter plane of the Rashba coupling $V_{R}$ and disorder strength $W$, which is shown in Fig. 3(b). We find that such a phase boundary is well correlated with the collapse of the bulk energy gap. In Fig. 4(a), we show that when $W=2.2 t$ is very close to $W_{c}=2.4 t$, localized bulk states are still well separated in energy from the conducting edge states appearing between $E \sim 0.0$ and $0.07 t$ with simple straight-line level crossing. With further increasing $W$ to $W=2.8 t$ slightly above $W_{c}$, the edge states and bulk states mix together, and level repulsion gaps show up, as indicated by the arrows in Fig. 4(b), which signals the presence of backward scattering and the collapse of the bulk energy gap.

Topologically invariant Chern number matrix. - At this stage, we have firmly established the connection between the QSHE in the model Eq. (1) and the presence of edge states within the bulk band gap. In the following, we examine whether there exists a topological characterization of the bulk states as in IQHE, which is responsible for the existence of the edge states.

Generally, in diagonalizing the Hamiltonian Eq. (1) one may introduce a generalized boundary condition $[5,17,18]$ for a 2D many-body wave function: $\Phi\left(\ldots, \mathbf{r}_{i_{\alpha}}+\right.$ $\left.\mathbf{L}_{j}, \ldots\right)=e^{i \theta_{j}^{\alpha}} \Phi\left(\ldots, \mathbf{r}_{i_{\alpha}}, \ldots\right)$, where $j=x, y$, and the system length vector $\mathbf{L}_{x}=\frac{N_{x}}{2} \mathbf{a}_{1}$ and $\mathbf{L}_{y}=N_{y} \mathbf{a}_{2}$, with $\mathbf{a}_{1}$ and $\mathbf{a}_{2}$ as two primitive vectors of the Bravais lattice. The twisted boundary condition is represented by $0 \leq \theta_{j}^{\alpha}<2 \pi$, where $\alpha=\uparrow$ and $\downarrow$ denotes the spin index. Through a unitary transformation $\Psi=$ $\exp \left[-i \sum_{\alpha} \sum_{i_{\alpha}}\left(\frac{\theta_{x}^{\alpha}}{L_{x}} x_{i_{\alpha}}+\frac{\theta_{y}^{\alpha}}{L_{y}} y_{i_{\alpha}}\right)\right] \Phi$, where the summation runs over all electrons of both spins, $\Psi$ becomes periodic on a torus. One can then define the topological Chern numbers as $[3,18]$
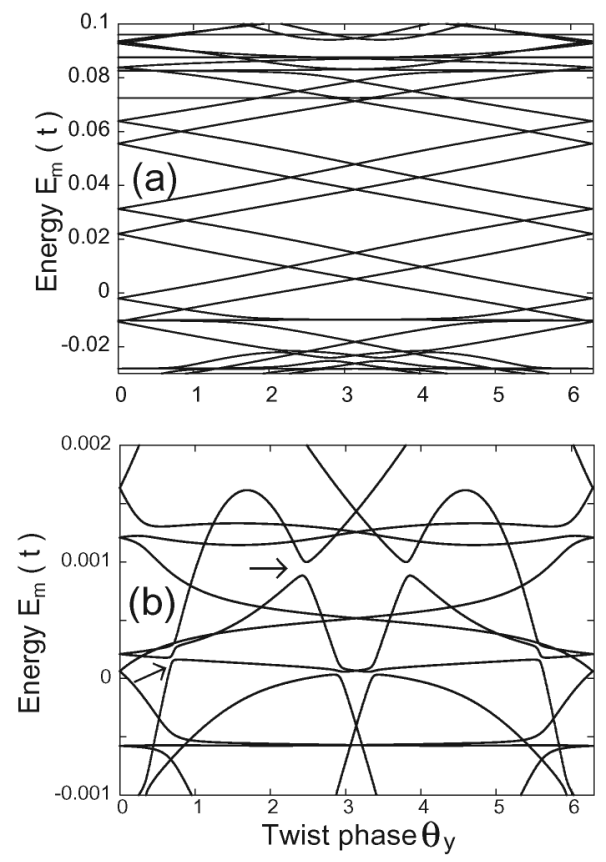

FIG. 4. (a) Level crossing below $W_{c}$ at $W=2.2 t$ in the energy region $0.0-0.07 t$. (b) The avoided level crossing (pointed by arrows) between edgelike states above $W_{c}$ at $W=2.8 t$ with changing $\theta_{y}$. Here, $N=240 \times 120, V_{\mathrm{SO}}$, and $V_{R}$ are the same as in Fig. 3(a) with $W_{c}=2.4 t$.

$$
C^{\alpha, \beta}=\frac{i}{4 \pi} \iint d \theta_{x}^{\alpha} d \theta_{y}^{\beta}\left[\left\langle\frac{\partial \Psi}{\partial \theta_{x}^{\alpha}} \mid \frac{\partial \Psi}{\partial \theta_{y}^{\beta}}\right\rangle-\left\langle\frac{\partial \Psi}{\partial \theta_{y}^{\beta}} \mid \frac{\partial \Psi}{\partial \theta_{x}^{\alpha}}\right\rangle\right],
$$

where the area integration is over a unit cell $0 \leq \theta_{x}^{\alpha}, \theta_{y}^{\beta} \leq$ $2 \pi$. With $\alpha, \beta=\uparrow, \downarrow, C^{\alpha, \beta}$ form a $2 \times 2$ CNM [18].

The many-body wave function $\Psi(\theta)$ is necessarily a smooth function of the boundary phase $\theta=\left\{\theta_{x}^{\alpha}, \theta_{y}^{\beta}\right\}$ when the energy gap remains. One can then prove the exact quantization of $C^{\alpha, \beta}$ by strictly following the argument of Thouless et al. [3]. Each topologically invariant matrix element $C^{\alpha, \beta}$ should remain unchanged until the energy gap collapses. Considering the simple case without the Rashba coupling term $\left(V_{R}=0\right)$, the only nonzero matrix elements are the diagonal ones: $C^{\alpha, \alpha}=\eta_{\alpha}$ with $\eta_{\uparrow}=$ $-\eta_{\downarrow}=1$ for $V_{\mathrm{SO}}>0$, which change sign with $V_{\mathrm{SO}}$. While the total charge Chern number $C_{c} \equiv \sum_{\alpha, \beta} C^{\alpha, \beta}$ (corresponding to the total charge Hall conductance of the system) [18] cancels out, the total spin-related Chern number is quantized to $C_{\mathrm{sc}} \equiv \sum_{\alpha, \beta} \alpha C^{\alpha, \beta}=2$, which represents the spin-Hall response when a common electric field for both spin components is imposed along the $\hat{\mathbf{y}}$ direction. In this spin decoupled limit, the quantized spin Chern number $C_{\mathrm{sc}}$ is associated with the SHC by $\sigma_{s H}=$ $C_{\mathrm{sc}}=2$ in units of $\frac{e}{4 \pi}$.

Now we turn on the Rashba coupling and numerically determine $C_{\text {sc }}$. This can be done either by calculating each $C^{\alpha, \beta}$ first or carrying out the integration in Eq. (2) for the opposite boundary condition along the $\hat{\mathbf{x}}$ direction (spin 


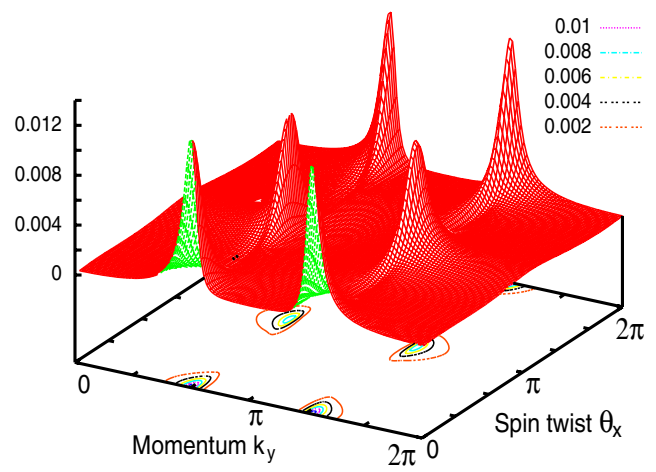

FIG. 5 (color online). Solid angle $\Omega_{j}$ vs spin twist $\theta_{x}$ and momentum $k_{y}$, which is meshed into $N_{\text {mesh }}=120 \times 120$ points for a pure system with $N=240 \times 240$ at $V_{\text {SO }}=0.1 t$ and $V_{R}=$ $0.3 t$. The total Berry phase $\sum_{j=1}^{N_{\text {mesh }}} \Omega_{j}=4 \pi$, and thus $C_{\mathrm{sc}}=2$.

twist $\left.\theta_{x}^{\uparrow}=-\theta_{x}^{\downarrow}=\theta_{x}\right)$ and the common one along the $\hat{\mathbf{y}}$ direction $\left(\theta_{y}^{\uparrow}=\theta_{y}^{\downarrow}=\theta_{y}\right)$ to directly obtain $C_{\mathrm{sc}}$. We choose the latter method in the following. The unit cell of the boundary phases is divided into $N_{\text {mesh }}=400$ to 14400 mesh points such that the integration in Eq. (2) is replaced by the sum of the solid angle $\Omega_{j}: C_{\mathrm{sc}}=\sum_{j} \frac{\Omega_{j}}{2 \pi}$. Here, $\Omega_{j}=$ $\arg \prod_{i}\left\langle\Psi_{j_{i}} \mid \Psi_{j_{i+1}}\right\rangle$, where $i=1-4$ (with $j_{5} \equiv j_{1}$ ) denote four mesh points at the $j$ th square of mesh patches in the $\theta$ space. Numerically it is verified that the computed $C_{\text {sc }}$ is well converged and insensitive to mesh sizes. We find that $C_{\mathrm{sc}}$ always remains quantized at $C_{\mathrm{sc}}=2$ before the band gap collapses at a critical Rashba coupling, say, $V_{R}^{c}=$ $0.33 t$ if $V_{\text {SO }}$ is fixed at $0.1 t$. We note that with a finite $V_{R}$ the spin becomes nonconserved, but it does not change the topological quantization as the latter is protected by the bulk energy gap. However, the nonconservation of spin is reflected in SHC, which becomes nonquantized as discussed before.

The total spin Chern number $C_{\mathrm{sc}}$ can be also expressed as the sum of the Berry phase of each eigenstate below the Fermi energy. Since along the $\hat{\mathbf{y}}$ direction a common boundary condition is imposed, which does not break the translational invariance of the system along this direction, one can calculate the contribution to $C_{\mathrm{sc}}$ of the eigenstates for a given momentum $k_{y}$ and formulate $C_{\mathrm{sc}}$ as an area integral over $k_{y}$ and $\theta_{x}$. Interestingly, at a larger $V_{R}=0.30 t$, the energy gap is about to collapse, and correspondingly the nonzero $\Omega_{j}$ becomes quite localized and concentrated at four points in the phase space $\left(0, \pm k_{y}^{*}\right)$ and $\left(\pi, \pm k_{y}^{*}\right)$, as shown in Fig. 5, where $k_{y}^{*}=\frac{2 \pi}{3}$ (it appears that there are six peaks simply because of the periodicity in $\theta_{x}$ ). As a matter of fact, we find that each peak in Fig. 5 carries about halfquantized topological charge by performing area integral around the sharp peak, while the rest of the area almost has no contribution to the Chern number $C_{\mathrm{sc}}$. With further increasing $V_{R}$, these half-quantized topological charges will mix and merge with the ones coming from the upper energy band (with opposite signs), when the band gap disappears and the two energy bands touch. For a pure sample, a step jump of the $C_{\mathrm{sc}}$ from 2 to 0 at a critical Rashba coupling $V_{R}^{c} \simeq 0.33 t$ is observed, corresponding to a quantum phase transition into a metallic state. The phase boundary for the topological quantized $C_{\mathrm{sc}}=2 \mathrm{QSHE}$ state has also been examined in the presence of disorder, where we find that the number of edge channels $N_{\text {edge }}$ [see Fig. 3(b)] has one-to-one correspondence to the spin Chern number $C_{\mathrm{sc}}$, and thus we identify the CNM as the topological origin of the nontrivial QSHE.

To summarize, we have numerically studied the topological description of a band insulator characterized by a spin Chern number $C_{\mathrm{sc}}= \pm 2$ and established its relation with a pair of ideal conducting edge states within the bulk band gap in an open system, which carry spin polarization and can pump spins from one edge to the other to result in QSHE. The spin Chern number $C_{\mathrm{sc}}$ remains precisely quantized until the band gap collapses at strong disorder or Rashba coupling, which distinguishes between two QSHE systems with opposite signs of SHC and thus further classifies a QSHE system with a nontrivial $Z_{2}$ index [9].

D. N. S. would like to thank D. Arovas, B. A. Bernevig, and S.-C. Zhang for stimulating discussions. This work is supported by No. ACS-PRF 41752-AC10, NSF Grant No. DMR-0307170 and No. DMR-0605696 (D. N.S.), the NSF (under MRSEC Grant No. DMR-0213706) at the Princeton Center for Complex Materials (F.D.M.H.), NSFC Grants No. 90403016 and No. 10374058 (Z. Y. W.), and a grant from the Robert A. Welch Foundation under Grant No. E-1146 (L. S.).

[1] For a review, see The Quantum Hall Effect, edited by R. E. Prange and S. M. Girvin (Springer-Verlag, Berlin, 1990).

[2] X. G. Wen and Q. Niu, Phys. Rev. B 41, 9377 (1990).

[3] D. J. Thouless et al., Phys. Rev. Lett. 49, 405 (1982).

[4] M. V. Berry, Proc. R. Soc. A 392, 45 (1984).

[5] Q. Niu, D. J. Thouless, and Y.S. Wu, Phys. Rev. B 31, 3372 (1985).

[6] F. D. M. Haldane, Phys. Rev. Lett. 93, 206602 (2004).

[7] D. N. Sheng et al., Phys. Rev. Lett. 90, 256802 (2003).

[8] F. D. M. Haldane, Phys. Rev. Lett. 61, 2015 (1988).

[9] C.L. Kane and E. J. Mele, Phys. Rev. Lett. 95, 146802 (2005); 95, 226801 (2005).

[10] L. Sheng, D. N. Sheng, C. S. Ting, and F. D. M. Haldane, Phys. Rev. Lett. 95, 136602 (2005).

[11] X.-L. Qi et al., cond-mat/0505308; B. A. Bernevig and S.-C. Zhang, Phys. Rev. Lett. 96, 106802 (2006); C. Wu et al., ibid. 96, 106401 (2006).

[12] S. Murakami, N. Nagaosa, and S. C. Zhang, Science 301, 1348 (2003); J. Sinova et al., Phys. Rev. Lett. 92, 126603 (2004).

[13] C. Xu and J.E. Moore, Phys. Rev. B 73, 045322 (2006).

[14] R. B. Laughlin, Phys. Rev. Lett. 50, 1395 (1983).

[15] K. S. Novoselov et al., Science 306, 666 (2004).

[16] D. N. Sheng et al., Phys. Rev. B 72, 153307 (2005).

[17] D. P. Arovas et al., Phys. Rev. Lett. 60, 619 (1988).

[18] D. N. Sheng, L. Balents, and Z. Wang, Phys. Rev. Lett. 91, 116802 (2003). 